\title{
Production and reporting of research evidence
}

When a new article is submitted for publication, scientific journal editors always wonder: What does this research mean? Does it add anything to scientific knowledge? This question is based on the fact that every year 160 billion dollars are invested in biomedical research, resulting in approximately one million scientific publications yearly. ${ }^{1}$

Expenditure in research and development is made up of revenue and capital (public and private) expenses in creative systematic work aimed at improving the level of knowledge, including knowledge about mankind, culture and society, and how such knowledge is used for new applications. Research and development includes basic research, applied research and experimental development.

In the United Kingdom, two thirds of resources are allocated to basic research, and less than $10 \%$, to treatment evaluation. While in the United States of America it is requested that treatment evaluation funding is independent from the industry; in Italy and Spain, independent research on new drug effects has been funded by a share of the taxes levied on drug promotion by the pharmaceutical industry. ${ }^{2}$

The United Nations Educational, Scientific and Cultural Organization (UNESCO) Institute for Statistics published that research and development expenses in Argentina were (as percentage of the GDP) $0.52 \%$ in 2009, while they were $1.08 \%$ in Brazil, and $0.39 \%$ in Chile. ${ }^{3}$

In addition, Argentina has seen a gradual and sustained rise in the number of scientific and technical publications, going from 3567 in 20032007 to 3665 in 2008-2012, an increase of $2.74 \%{ }^{4}$

As a result, research has become an enterprise, a financial machine for some countries, a necessary step in the road towards economic growth. However, the dissemination of research results has not been quite encouraging.

A systematic review of 709 follow-up studies of research published in abstracts estimated that the rate of publication of full reports after 9 years was only $53 \% .^{5}$

Also, new investigations are usually unnecessary and poorly designed, thus wasting resources. ${ }^{6}$ Biased under-publication or duplicated studies constitute scientific and ethical misconduct, and the public is being gradually warned about this situation, especially due to several disclosures regarding evidence on how serious adverse effects of treatments had been concealed. ${ }^{7}$

In general, studies with discouraging results have a lesser chance of being readily published, a higher probability of being disseminated in publications of a poor scientific level, and less likelihood of abstracts becoming full papers. ${ }^{5}$

An advance made in the past decade has been the invitation to include all clinical trials in a public registry due to the influence of several organizations, such as the World Health Organization (WHO), the World Medical Association (through the latest review of the Declaration of Helsinki) and the International Committee of Medical Journal Editors (ICMJE), thus achieving certain progress.

Although the WHO's International Clinical Trials Registry Platform has improved transparency and social participation in research, public access to all the results of all research studies is still wishful thinking, particularly because it has been resisted by certain funders and researchers.

It has been estimated that $85 \%$ of research is ineffective and results in resource wasting, with deficiencies in four areas of interest: ${ }^{6}$

1. Is the investigation question relevant for physicians or patients?

2. Are the design and methods appropriate?

3. Are all results accessible?

4. Is the study clinically significant and unbiased?

Since investigation goes through all these stages, the loss is cumulative. It has been estimated that from an initial loss of $50 \%$, stages 2,3 and 4 end with a loss of more than $85 \%$.

This implies that the proceeds of important financial resources invested in research are lost every year due to correctable errors, and this can be applied to any type of investigation.

Due to the different problems in each stage of the production and reporting, there is no single solution. Some of the recommendations for each of the stages described herein include: ${ }^{6}$

1. Promoting patient and physician participation in the design of investigation agendas and in the formulation of specific questions. Encouraging courses of "scientific initiation" and the critical appraisal of systematic reviews.

2. Promoting research methodology courses. 
Increasing the number of health research "methodologists." Promoting the support from funding organizations for the development of systematic reviews on the evidence. For example, the NIHR's Health Technology Assessment Programme in the United Kingdom requests systematic reviews before providing funds for studies, publishes its research in the web site (http://www.hta. ac.uk/about/index.shtml), and since 2006 it allows open access to all new protocols. Editors should request a thorough review of previous similar studies.

3. Requesting, through incentives or standards, the registration and reporting of all clinical trial protocols before they begin. Supporting open access to all results once research is completed.

4. Encouraging authors and editors to receive training on how to report and evaluate studies, for example, the CONSORT and STARD statements (http://www.equator-network. org). In addition topeer reviews, include user and methodologist reviews, because many journals favor the "contribution to knowledge" aspect over methods and their application. Supporting open access to repositories, separately from publications, where physicians and researchers can obtain details on the treatments, tests and instruments used due to the space restrictions of journals.

Finally, we should ask ourselves and ponder about how we got to this point and how we could restructure and restore the procedures to conduct and compensate investigation. First of all, we should have in mind what the true aim of investigation is. Then we should elucidate the means to decide what type of research is required and the impact it will have. Academic institutions should allow compensations to be paid, and compensate researchers in the long run therefore favoring an easier access to the actual and important impact of its investigation. Lastly, researchers should strive more to defend the means to conduct investigations that benefit the population's health worldwide and not only as a component of economic policies. ${ }^{1}$

\section{Carlos Grandi, M.D.}

Editorial Board

http:/ /dx.doi.org/10.5546/aap.2013.276

1. Editorial. What is the purpose of medical research. The Lancet 2013;381:347.

2. Garattini S, Chalmers I. Patients and the public deserve big changes in evaluation of drugs. BMJ 2009;338:804-6.

3. Banco Mundial. Gasto en investigación y desarrollo (\% del PIB). [Accessed on: march 4, 2013]. Available at http:// datos.bancomundial.org/indicador/GB.XPD.RSDV. GD.ZS.

4 Fundación Nacional de la Ciencia. Indicadores del desarrollo mundial. Artículos en publicaciones científicas y técnicas. [Accessed on: march 4, 2013]. Available at http://datos.bancomundial.org/indicador/IP.JRN.ARTC. SCcountries.

5. Scherer RW, Langenberg P, von Elm E. Full publication of results initially presented in abstracts. Cochrane Database Syst Rev 2007;2:MR000005.

6. Chalmers I, Glasziou P. Avoidable waste in the production and reporting of research evidence. Lancet 2009;374:86-9.

7. Chalmers I. From optimism to disillusion about commitment to transparency in the medico-industrial complex. J R Soc Med 2006;99:337-41.

\section{Allergic rhinitis and pulmonary function}

Asthma is the most common chronic disease among children creating a substantial burden on health systems. Epidemiological studies, such as the ISAAC, have provided more accurate evidence on the magnitude of this condition. ${ }^{1}$ Allergic rhinitis is also a very common condition, but given its wide clinical spectrum and lesser severity, it is less known than asthma. Although evidence has been contributed on the possible link between both conditions, the real relationship between asthma and allergic rhinitis is still being discussed.

Allergic rhinitis is highly common among asthma patients and there is evidence regarding its impact on asthma. The control of rhinitis in asthma patients leads to a better management of asthma (by reducing the risk of hospitalization and visits to the emergency department); for this reason, rhinitis is considered one of the main comorbidities, together with gastroesophageal reflux, obesity and smoking. From a different approach, the presence of rhinitis could be considered a risk factor for the further development of asthma. ${ }^{2}$

The mechanism that relates both conditions is not clear, although it is highly likely that its association is not merely epidemiological and is related to common pathophysiological processes. ${ }^{3}$ 
Studies on the role of innate immunity on the physiopathogenesis of allergic diseases and atopic asthma provide interesting data in this regard. ${ }^{4}$

Given this likely association, it is interesting to identify elements to better individualize patients with rhinitis who, in spite of not having asthma symptoms, may have pulmonary function disturbances, quite likely at a sub-clinical level of the disease.

There is evidence that patients with allergic rhinitis might have a high prevalence of bronchial hiperresponsiveness and disturbances in the functional testing of breathing. ${ }^{5}$ Given that the prevalence of allergic rhinitis may have more geographic variations than that of asthma, ${ }^{6}$ local and regional studies are more significant.

In this issue of ArchivosArgentinos de Pediatría Ianiero, et al. look into the results of pulmonary function tests among children and adolescents with allergic rhinitis without a diagnosis of asthma. ${ }^{7}$ The authors approach the potential magnitude of this link, and also explore the possible relationship with other elements that could be used in the daily practice. Therefore, they identify a likely association between altered values for forced spirometry and an increased peripheral blood eosinophil count, which would account for a pathophysiological mechanism common with the atopic asthma phenotype in these cases. In addition, authors have revived the findings of a marginal association between altered pulmonary function tests and body mass index (BMI). Although the relationship between asthma and obesity has been extensively studied, ${ }^{8}$ the influence of overweight on pulmonary function should be considered in greater depth, ${ }^{9}$ especially in children without morbid obesity. ${ }^{10}$

It is desirable that this initiative be deepened by means of studies with a sample size and design that allow to precisely estimate the prevalence of disturbances of the pulmonary function among children with allergic rhinitis and its association with obesity taking into consideration the variations in body mass index (BMI) in the studied period of life.

Fernando Ferrero, MD

Hospital General de Niños Pedro de Elizalde, Buenos Aires

http:/ /dx.doi.org/10.5546/aap.2013.277

\section{REFERENCES}

1. Lai CK, Beasley R, Crane J, Foliaki S, et al. International Study of Asthma and Allergies in Childhood Phase Three Study Group. Global variation in the prevalence and severity of asthma symptoms: phase three of the International Study of Asthma and Allergies in Childhood (ISAAC). Thorax 2009 ; 64(6):476-83.

2. Thomas M. Allergic rhinitis: evidence for impact on asthma. BMC Pulm Med 2006; 6 Suppl 1:S4.

3. Jeffery PK, Haahtela T. Allergic rhinitis and asthma: inflammation in a one-airway condition. BMC PulmMed 2006; 6 Suppl 1:S5.

4. Holgate ST. Innate and adaptive immune responses in asthma. NatMed 2012; 18(5):673-83.

5. Ciprandi G, Tosca MA, Signori A, Cirillo I. Bronchial hyperreactivity in patients with allergic rhinitis: forced expiratory flow between 25 and $75 \%$ of vital capacity might be a predictive factor. AllergyAsthmaProc 2011; 32(2):4-8.

6. Katelaris $\mathrm{CH}$, Lee BW, Potter PC, Maspero JF, et al. Prevalence and diversity of allergic rhinitis in regions of the world beyond Europe and North America. ClinExpAllergy 2012; 42(2):186-207.

7. Ianiero L, Saranz R, Lozano NA, Lozano A, Sasia L, Ramirez M, Cuestas E. Análisis de la curva flujo-volumen en niños y adolescentes con rinitis alérgica sin asma. Arch Argent Pediatr 2013; 111(4):322-27.

8. Gold DR, Damokosh AI, Dockery DW, Berkey CS. Bodymass index as a predictor of incident asthma in a prospective cohort of children. PediatrPulmonol. 2003; 36(6):514-21.

9. Littleton SW. Impact of obesity on respiratory function. Respirology 2012; 17(1):43-9.

10. Boran P, Tokuc G, Pisgin B, Oktem S, Yegin Z, Bostan O. Impact of obesity on ventilatory function. J Pediatr (Rio J). 2007; 83(2):171-6.

\section{Smoking among pediatric residents in Argentina}

In 2007, a research trial, the TAMARA study, was conducted in Argentina regarding smoking among physicians. Results published by Zylbersztejn, et al. ${ }^{1}$ showed that out of 6497 surveyed physicians, $30 \%$ smoked and $22.4 \%$ were former smokers. The higher smoking prevalence was observed among emergency medicine and surgical specialty physicians showing an addictive behavioral profile. Smoking physicians advised patients less on smoking cessation. However, those who had been trained on this topic while studying at college were more active in addressing smoking cessation.

In this issue, Gigliotti, et al. (see page 315) show 
smoking prevalence among pediatric residents in Argentina, according to a survey administered in $2011(20.1 \%)$. When compared to data from $2002^{2}$ smoking prevalence has declined but not significantly (2002: 22.1\%) and currently, a higher rate of resident physicians take an active role in helping smokers quit.

Smoking implies the consumption of psychoactive substances, such as nicotine, which is considered a legal drug with negative consequences on health. The regular use of this type of substances results in dependence with a great impact on personal life: a craving to smoke, withdrawal symptoms, need to increase use, time and resources to buy tobacco, limitation of their social circle due to the increasing rejection of certain sectors to passive smoking and the known consequences of smoking on a person's health and on the health of others.

The use of psychoactive substances is related to general factors, including a genetic predisposition, psychosocial and biological aspects, family history, and specific factors, such as undergoing highly stressful situations.

When smoking is discussed among physicians, the question that always comes up is: knowing the harmful effects of smoking, how could you explain this incoherent behavior? The explanation is obvious. Physicians have the same conflicts, the same difficulties to cope with problems, the same likelihood of falling into an addiction as well as risk factors known to be associated with addictive behavior as every other people.

But the impact of such inconsistency is even more significant given the role played by physicians before their patients, as models of health behaviors. ${ }^{3}$ Smoking physicians tend to advise patients less on smoking cessation, ${ }^{4}$ and this is one more hurdle in the compliance of the commitment made by many countries, including Argentina, in the Framework Convention on Tobacco Control.

In 1998, in Europe, the College of Physicians in Barcelona developed the Program of Comprehensive Care for Sick Physicians (Programa de Atención Integral al Médico Enfermo, PAIME) with psychological problems or addictive behaviors. Occupational stress resulting from the accumulated working time and scarce resources has been considered as a risk factor for the development of psychological problems or addictive behavior and consequently, malpractice. It is estimated that 1 out of 10 physicians suffers from some of these disorders.
Within this Program, the most common causes for seeking care include mental disorders (67\%), alcohol abuse (16\%), dual disorders (10\%) and drug use $(7 \%)$. Physicians in training account for $9 \%$ of those who receive treatment as part of the program, and the number is increasing. ${ }^{5}$

It is interesting to see how admission to the Program is the initiative of the affected physician in half of the cases, while the other half of physicians enters the Program referred by a colleague, a member of the family, the boss, or a member of the occupational risk department. The health of physicians is considered a responsibility of the College of Physicians and of the community as a whole. The approach to this problem is to help addicts and be committed to their professional environment.

A study conducted in India ${ }^{6}$ has disclosed another alarming data. A survey administered to students of two schools of medicine in Southern India showed that the number of smokers increased from $13.6 \%$ to $26.1 \%$, and that the number of alcohol users increased from $19.3 \%$ to $43.8 \%$ while attending the schools of medicine. Besides, over the same period, the number of students who did some kind of physical activity declined from $43.2 \%$ to $24.4 \%$, and their eating habits became less healthy. Disseminating healthy measures aimed at reducing the risk of adult chronic conditions is one of the responsibilities of physicians, and it is also part of patients' expectations; therefore, these aspects should neither be overlooked nor get worse during the professional training period.

In addition to detecting this problem, it is about time to consider strategies for the early prevention of smoking and the promotion of healthier lifestyles, starting in school years but with special emphasis on the period of professional and postgraduate training, when other factors predisposing to addictions usually become much more apparent.

Norma Rossato, M.D. Associate Editor

http:/ /dx.doi.org/10.5546/aap.2013.278

1. Zylbersztejn HM, Cardone A, Vainstein N, Mulassi A, et al. Tabaquismo en médicos de la República Argentina. Estudio TAMARA. Rev Argent Cardiol 2007;75:109-116.

2. Ferrero F, Castaños C, Durán P, Blengini MT. Prevalencia del consumo de tabaco en médicos residentes de pediatría en Argentina. Rev Panam Salud Pública 2004;15(6):395-9. 
3. Hanks DT,Antonuccio DO. Physician modeling influences on patient smokers. Addict Behav 1987;12(3):257-62.

4. Fonseca M, Fleitas G, Tamborero G, Benejam M, et al. Lifestyles of primary care physicians: Perception and implications on cardiovascular prevention. Semergen 2013 Apr 11. pii: S1138-3593(13)00016-6.
5. Consejo General de Colegios Oficiales de Médicos de España. PAIME: Programa de Atención Integral del Médico Enfermo. Disponible: https://www.cgcom.es/ noticias/2013/05/13_05_23_paime_estudio.

6. JP Majra. Do Our Medical Colleges Inculcate HealthPromoting Lifestyle Among Medical Students: A Pilot Study from Two Medical Colleges from Southern India. Int J Prev Med 2013 April; 4(4): 425-429. 